BROOKHAVEN NATIONAL LABORATORY

Associated Universities, Inc.

Upton, New York

\title{
ACCELERATOR DEPARTMENT
}

Informal Report

HIGH TRANSVERSE MOMENTUM AT ISABELLE

L. M. Lederman

Columbia University

February 1974

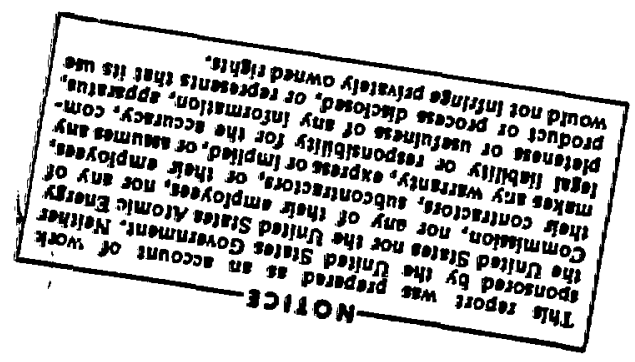

NOTICE

This report was prepared as an account of work sponsored by the United States Government. Neither the United States nor the United States Atomic Energy Commission, nor any of their employees, nor any of their contractors, subcontractors, or their employees, makes any warranty, express or implied, or assumes any legal liability or responsibility for the accuracy, completeness or usefulness of any information, apparatus, product or process disclosed, or represents that its use would not infringe privately owned rights.

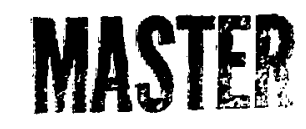


Figh Transverse Momentum at Isabelle

I. M. Lederman

There is a pervasive impression in high energy physics that high transverse momentum is somehow related to physics of small distances. This can be clearly demonstrated in electromagnetic interactions but is a much more diffuse considaration in hadron physics. However, the collective "hunch" that high $\mathbf{P}_{\mathbf{T}}$ hadronic plienomena probe deeply into the fundamental structure of hadrons is very strong: the surprisingly large yield observed at ISR and NAL is surely an important incentive to "go to higher $\varepsilon^{\prime \prime}$. In this case, it is similar to the rising tolal cross sections - physics is constrainsel to wait for the rossibility of fol lowing up on a tantalizing hint.

The present data on high $P_{T}$ hadronic phenomena may be summarized as follows:

i) Inclusive pion production: ${ }^{1}$

$E \frac{a^{3} \sigma}{a^{3} p}=190 e^{-7.1 P_{T}}+\frac{B e^{-26 \cdot P_{T} / \sqrt{3}}}{\left(P_{T}{ }^{2}+1\right)^{4}}(x \geqslant 0)$

This is a reasonable fit to al.1 the $\pi^{\circ}$ and \pm data obtained at ISR and accounts (within a factor of 3) also for observations at NAI $^{2}$ i.e.. from $\sqrt{s}=19$ to $\sqrt{s}=61$ GeV. The first term is the well known s-independent steep exponential, typical of the large fraction of the hadronic total cross section. The denominator of the second term is chosen to give a smooth fit to data at lower $P_{\text {T }}$. 


\section{ii) Correlations:}

It is known that charged particle multiplicities increase with the $P_{T}$ of the observed $\pi^{\circ}$ when these are in the opposite hemisphere. High $P_{T}$ pions are also associated with charged tracks on the same side but in a $P_{T}$ independent way. J.t is also known that a high $P_{T} \pi^{\circ}$ has some correlation $\left(\rho \sim 180^{\circ}\right.$ ) with clusters of charged tracks but that this correlation does not dominate the process. $3^{\circ}$ pinaliy, there is strong correlation between pairs of high $\psi_{T} \pi^{0} \cdot s$ in opposite hemispheres. 3

\section{iii) Quantum numbers:}

To date only $p-p$ and p-nuclear collisions ${ }^{2}$ have been observed. Outgoing high $P_{T}$ protons, kaons, and anti-protons

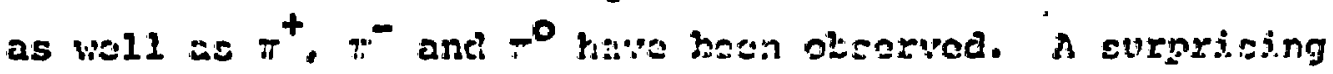
yield of protons have been observed in the NAL experiment. Issues which are not yet settled have to do with the following:

i) How is the transverse momentum balanced when one observed one large $P_{T}$ object?

ii) What is the relative probability for high $P_{T}$ to be observed in clusters of $P_{T}=\sum_{i} P_{T_{i}}$ vs. all $P_{T}$ invested in a single particle?

iii) What changes when pp collisions are replaced by $\pi p, \bar{p}$ ?

iv) What are the quantum numbers of the outgoing clusters, if any?

v) If clusters, what are the dynamics of cluster production?

vi) What happens in detail at higher values of $P_{T}$ for $s \rightarrow \infty$ ? Does the $P_{T}{ }^{-8}$ persist or does the curve again 
"break" into a flatter behavior?

vii) Where are the "kinematic boundaries" i.e.. are data for $P_{T}$ - $s$ ouppressed by leading particle effects, i.e. the need for the bombarding particles to continue their initial motions?

Many of the above questions are motivated by curxent models which may or may not be relevant in the future. 4,5 However, Isabelle i.e. $s \gg 3000 \mathrm{Gev}^{2}$ may well be essential in order to clarify these many questions. We concentrate on those which are not likely to be illuminated in the coming fuller development of ISR, NAL, and CERN II.

1. Jets. We note here that the available $s$ at ISR is marginal for the study of jet structure. Assume a multipljejty of $n$ partin? fes for a jet of $\mathrm{zP}_{\mathrm{rr}}=\mathbb{P}_{\mathrm{w}}$. The opening angle of a Bjorken-style jet is

$$
\theta \sim 0.3 \mathrm{n} / \overline{\mathrm{P}}_{\mathrm{T}}
$$

and for this to stand out cleanly enough for its dynamics (i.e. outgoing parton motion) to be differentiated from the background, we need

$$
\theta<0.3
$$

and the lowest component of $P_{T^{\prime}} P_{T_{i}}$ to be large i.e. $>2 \mathrm{GeV} / \mathrm{C}$.

$$
\text { Using } n \sim 3 \text { en } \bar{P}_{T} \text { lan irrefutable equation at this }
$$
writing:), we find

$$
\overline{\mathbf{P}}_{\mathrm{T}}>10 \mathrm{GeV} / \mathrm{C} \text {. }
$$


Ising single particle production as the oniy guide we have, the yield at the ISR is very low -- using the equation (1). it is higher by two orders of magnitude at Isabelle. 2. The study of jets of $P_{T} \geq 10 \mathrm{GeV} / \mathrm{c}$ means an $x_{\perp} \geq$ 0.4 at the ISR. This is getting rather close to the kinematic boundary at $x_{1}=1$ and in fact may already be constrained by the leading particle effects discussed above. Thus even if luminosity problems are overcome, the limited s-problem will persist.

3. The issue of the $\mathbf{s}$ dependence, the $P_{T}$ dependence and the approach to scaling will also require a much higher value of than is now available.

These may well be among the most crucial issues: the phonomena illuritrston hy Fr. (1) expnsos, for $P_{T} \geq 10 \mathrm{GeV} / \mathrm{C}$ a domain where the strong interaction becomes weak. Inaced, the original expectation ${ }^{4}$ of a scaling regime:

$$
E \frac{d^{3} o}{d p^{3}}=\frac{A}{P_{T}^{4}} F\left(\frac{P_{p}}{\sqrt{s}}, \theta\right) .
$$

was based on the plausibility that at least, $A \sim \alpha^{2}$.

It is seen that a weak parton-parton interaction would also give rise to (2) with comparable coefficient. (This can be obtained from the cve theorem and a popular choice of $W$ mass.) for $P_{T} \sim 10 \mathrm{GeV}$, the intensities of the strong term (1) and the pointlike weak (e.m.) term become comparable, assuming similar structure functions, $F \sim e^{-B x_{1}} \cdot T h: 3 s$ the region of $P_{T}>10 \mathrm{GeV} / \mathrm{c}$ becomes a domain where the 
conventional hierarchy of forces breaks down - all are comparable and interference effects may appear and eventually damp out. The predictions of (1) and (2) are displayed in Fig. I and give some indication of the need for an asymptotic s-accelerator. Models for the transition from regime (1) to regime (2) have recently been given by Bander et al 6 and by T.D. Lee. 7

In this connection, it is interesting to note that there has for some time been evidence for high $P_{T}$ events in cosmic ray observations. ${ }^{B}$ If these are correctly interpreted, the implication is for a dramatically different s-dependence, Halzen and Luthe ${ }^{9}$ have made a fit to ISR data, making explicit use of a scale breaking mass by replacing the variable, $x_{1}=2 P_{2} / \sqrt{ } \mathrm{B}$ by

$$
x_{t}^{\prime}=\frac{2 P_{T}}{\sqrt{s+m^{2}}} .
$$

In this way, they can preserve a $p^{-4}$ behavior and sti.l.1 evolve to scale invariance at $\sqrt{s} \gg \mathrm{m}$. Their fit gives $\mathrm{m} \equiv 30 \mathrm{GeV}$ and a rousing 3 orders of magnitude more cross section for Isabelle at $P_{\text {T }} \geq 10 \mathrm{GeV} / \mathrm{c}$ :

What $k$ ind of observations can one carry out in the new domain? One would surely study the change in behavior of correlations, quantum numbers, etc. Ratios of outgoing leptons to hadrons now become of crucial interest. Search for violations of discrete symmetries become necessary. For example, outgoing high $P_{\mathrm{T}}$ pion pairs may give rise to a finite expectation value for the term: 


$$
\overrightarrow{\mathbf{P}}_{+} \times \overrightarrow{\mathbf{P}}_{-} \cdot \overrightarrow{\mathbf{p}}_{\text {beam }}
$$

where $\overrightarrow{\mathrm{P}}_{\text {beam }}$ is defined as that direction giving a positive sign for $\overrightarrow{\mathrm{p}}_{\text {beam }} ;\left(\overrightarrow{\mathrm{p}}_{+}+\overrightarrow{\mathrm{p}}_{-}\right)$. Longitudinal polarization of protons, $\Lambda^{O_{1}}$,etc. must clearly distinguish direct production from secondaries of weak decay. A premiun is now placed on the ability of Isabelle to store deuterons in order to observe pd and da collisions to facilitate tests of charge independence.

A viable experimental approach to the above will almost certainly: involve several different kinds of arrangements. The universal proposal that will solve all problems isill. at the least, succeed in re-creating the dinosaur. One fairly missive approach might collect $2 \pi$ solid angle in axjmutit ind a bolar alikise of $50: 45^{\circ}$. It would stirround the interaction region with a superconducting solenoid having a radius of $\sim 2 \mathrm{~m}, H=15 \mathrm{kG}$, yielding a $\Delta P_{T} / P_{T} \sim 6 \%$ at $50 \mathrm{GeV} / \mathrm{c}$. The very small cross section of the interaction diamond helps very much in azimuthal reconstruction.

Assuming an interaction rate of $10^{7} / \mathrm{sec}\left(\mathrm{L} \sim 2 \times 10^{33}\right)$, each wire of a drift chamber ensemble needs to handle $\sim 1 c^{5} / \mathrm{sec}$.

Outside of the a 1 rad. length thick superconducting coils. some sort of $\pi^{\circ}$ and $e, \gamma$ spectrometer is needed. Such an arrangement could address many of the important issues enumerated above - but not all. The next few years of exploitation of this field may confirm the present 
thesis that much higher energy will be required to enable an incisive exploration of this new field. The possibility exists that this new field could play a key role in resolving the puzzle of the hierarchy of forces. 
REFERENCES

1 Blumenfeld et al. Phys. Lett. 46B, 471 (1973. Alpex et al, Phys. Lett. 46B, 265 (1973). The data of these groups is combined and a "hand-fit" obtained which favors the low $P_{T}$ normalization.

2 J. Cronin et al. Phys. Rev. Lett. 31. 1426 (1973). D. Carey et a1. Phys. Rev. Lett. 12, 24 (1974).

3 Busser et al, CCR Group Report to Vancierbilt Conference (1973); also P. Grannis (Pisa-Stony Brook Group) private communication.

4 Berman, Bjorken, Kogut, Phys. Rev. D4, 3388 (1971).

5 Blankenbecker, Brodsky, Gunion, Phys. Lett. 42B, 461 (1972).

6

Panes: ct: al

7 T.D. Lee, private communication.

8 McCuskar. Phys. Rev. 177, 1902 (1969).

9 F. Kalzen, J. Luthe, Univ. of Wisconsin, preprint December (1973). 
